\title{
Bosonization of the $Q=0$ continuum of Dirac fermions
}

\author{
Sebastian Mantilla and Inti Sodemann \\ Max-Planck Institute for the Physics of Complex Systems, D-01187 Dresden, Germany
}

(Received 4 June 2020; accepted 11 August 2020; published 8 September 2020)

\begin{abstract}
We develop a bosonization formalism that captures nonperturbatively the interaction effects on the $\mathbf{Q}=0$ continuum of excitations of nodal fermions above one dimension. Our approach is a natural extension of the classic bosonization scheme for higher dimensional Fermi surfaces to include the $\mathbf{Q}=0$ neutral excitations that would be absent in a single-band system. The problem is reduced to solving a boson bilinear Hamiltonian. We establish a rigorous microscopic footing for this approach by showing that the solution of such boson bilinear Hamiltonian is exactly equivalent to performing the infinite sum of Feynman diagrams associated with the Kadanoff-Baym particle-hole propagator that arises from the self-consistent Hartree-Fock approximation to the single-particle Green's function. We apply this machinery to compute the interaction corrections to the optical conductivity of two-dimensional Dirac fermions with Coulomb interactions reproducing the results of perturbative renormalization group at weak coupling and extending them to the strong-coupling regime.
\end{abstract}

DOI: 10.1103/PhysRevB.102.121103

Introduction. The remarkable success of bosonization in capturing the nonperturbative properties of interacting fermions in one dimension [1] has long motivated the quest for extensions of this program to higher dimensions. One major such enterprise has been the development of higher dimensional bosonization of Fermi surfaces [2-6]. In this approach, particle-hole creation operators of a given total momentum $\mathbf{Q}, c_{\mathbf{k}+\mathbf{Q} / 2}^{\dagger} c_{\mathbf{k}-\mathbf{Q} / 2}^{\dagger}$, are promoted to bosonic creation operators with a commutator that is approximated as a number. The resulting bosonized Hamiltonian only couples bosonic modes with momentum $\mathbf{Q}$ to bosons with either $+\mathbf{Q}$ or $-\mathbf{Q}$. Namely, there is zero amplitude for a particle-hole pair with momentum $\mathbf{Q}$ to transition into two particle-hole pairs with momenta $\mathbf{Q}_{1,2}$ and $\mathbf{Q}=\mathbf{Q}_{1}+\mathbf{Q}_{2}$. The only allowed processes are for the particle-hole pair with momentum $\mathbf{Q}$ to scatter into another one with the same $\mathbf{Q}$, or to create pairs of particle-hole pairs with momentum $+\mathbf{Q}$ and $-\mathbf{Q}$ [see, e.g., Eq. (7.1) in Ref. [7]]. This assumption of separability of Hilbert spaces of particle-hole pairs with different $|\mathbf{Q}|$, lies at the heart of the higher dimensional bosonization approach to Fermi surfaces and it is believed to be an asymptotically correct description of particle-hole excitations of Landau Fermi liquids at small $|\mathbf{Q}|$.

Ordinary single-band Fermi liquids do not have low-energy particle-hole excitations with total momentum $\mathbf{Q}=0$ and therefore this sector does not appear in the conventional problem of bosonization of Fermi surfaces. In contrast nodal semimetals in which the Fermi surface shrinks to a point,

Published by the American Physical Society under the terms of the Creative Commons Attribution 4.0 International license. Further distribution of this work must maintain attribution to the author(s) and the published article's title, journal citation, and DOI. Open access publication funded by the Max Planck Society. have a nontrivial set of gapless optical $\mathbf{Q}=0$ particle-hole excitations. The central purpose of the present study is to develop a systematic bosonization approach to this sector for gapless semimetals. For concreteness we will discuss on two-dimensional (2D) massless Dirac fermions, such as those appearing in graphene and the surface of three-dimensional topological insulators, but our ideas can be naturally extended to other cases and higher dimensions. To describe such excitations, we will borrow the central assumption of the bosonization approach of Fermi surfaces, namely, that such optical particle-hole pairs are decoupled from the particlehole pairs of finite momentum $\mathbf{Q}$. We expect this simplification to be justified at low energies in phases which are adiabatically related to free fermions, in a similar sense to how such decoupling allows one to describe Fermi liquids which are adiabatically related to free fermions in the higher dimensional bosonization of Fermi surfaces. We will, however, establish an explicit microscopic connection between our bosonization approach and the conventional Feynman diagrammatic perturbation theory that demonstrates the validity of this central assumption of our approach. Specifically, we will prove that the solution of our effective bosonic Hamiltonian for the optical particle-hole pairs is exactly equivalent to the self-consistent Kadanoff-Baym resummation [8,9] of the particle-hole propagator at $\mathbf{Q}=0$, associated with the selfconsistent Hartree-Fock approximation to the single particleparticle Green's function.

As an application of our approach we will compute the interaction corrections to the optical conductivity of 2D Dirac fermions with Coulomb interactions, whose strength is parametrized by the effective fine-structure constant $\alpha=$ $e^{2} / \epsilon v$, where $v$ is the velocity of the Dirac fermions and $\epsilon$ the dielectric constant of the surrounding medium. This optical conductivity at low energies is determined by fundamental constants of nature, and given by $\sigma_{0}=e^{2} / 16 \hbar$ per Dirac cone $[10,11]$. Its zero frequency limit is not expected to be 
renormalized by interactions, but, Coulomb interactions can produce a slow flow as a function of frequency to such value and a nontrivial nonanalytic frequency dependence at low energies. Early perturbative calculations of such corrections were in mutual disagreement $[12,13]$, but subsequent studies [14-20] validated the result of Ref. [12]. As we will see, our approach recovers the perturbative results of Ref. [12] at small interactions and extends them nonperturbatively to finite $\alpha$. Nonperturbative attempts to understand the effects of Coulomb interactions in the optical conductivity of Dirac fermions have been scarce. A quantum Monte Carlo effort [21] to compute the optical conductivity concluded that interaction corrections remain rather small even at $\alpha \sim 2$. Our analysis will also support this conclusion, which is broadly in agreement with experiments that have found values close to that for noninteracting fermions [22-24].

Effective Hamiltonian and Hilbert space. The microscopic Hamiltonian is $(\hbar=1)$

$$
\begin{aligned}
H= & v \sum_{\mathbf{k}, \sigma, \sigma^{\prime}} \psi_{\mathbf{k}, \sigma}^{\dagger}\left(\mathbf{k} \cdot \sigma_{\sigma \sigma^{\prime}}\right) \psi_{\mathbf{k}, \sigma^{\prime}}^{\dagger} \\
& +\frac{1}{2 A} \sum_{\mathbf{k} \mathbf{k}^{\prime}} \sum_{\sigma \sigma^{\prime}} V_{\mathbf{q}} \psi_{\mathbf{k}^{\prime}+\mathbf{q}, \sigma^{\prime}}^{\dagger} \psi_{\mathbf{k}-\mathbf{q}, \sigma}^{\dagger} \psi_{\mathbf{k}, \sigma}^{\dagger} \psi_{\mathbf{k}^{\prime}, \sigma^{\prime}}^{\dagger},
\end{aligned}
$$

where $A$ is the system area, and $V_{\mathbf{q}}$ is the Fourier transform of the interaction potential. It is convenient to imagine the fermions moving in a $2 \mathrm{D}$ torus so that its momentum is quantized on a lattice. In this momentum lattice the complete many-body Hilbert space is a tensor product of empty, singly, and doubly occupied states:

$$
\mathcal{H}=\bigotimes_{\mathbf{k}}\left(|0\rangle_{\mathbf{k}} \oplus|\uparrow\rangle_{\mathbf{k}} \oplus|\downarrow\rangle_{\mathbf{k}} \oplus|\uparrow \downarrow\rangle_{\mathbf{k}}\right)
$$

The kinetic term in Eq. (1) produces no fluctuations between the occupancy of the momentum sites, and favors a ground state with singly occupied states with a suitably oriented spin in the form of vortex around the Dirac point [see Fig. 1(a)]. The interactions are pair hopping terms with a finite amplitude to induce transitions into states with doubly occupied sites and empty sites. Crucially, the subspace of the Hilbert space with singly occupied sites is equivalent to the space of particle-hole pairs with zero total momentum, $\mathbf{Q}=0$, while those states with doubly occupied and empty sites contain particle-hole excitations of finite momentum $\mathbf{Q}$. Therefore, following the spirit of higher dimensional bosonization, we project the Hamiltonian in Eq. (1) onto the Hilbert space of singly occupied sites in the momentum lattice, depicted in Fig. 1(b). This Hilbert space contains a spin-1/2 at each momentum site:

$$
\mathcal{H}_{\text {single }}=\bigotimes_{\mathbf{k}}\left(|\uparrow\rangle_{\mathbf{k}} \oplus|\downarrow\rangle_{\mathbf{k}}\right),
$$

and the projection of the Hamiltonian from Eq. (1) leads to the following Heisenberg model:

$$
\mathcal{P} H \mathcal{P}=\sum_{\mathbf{k}} v \mathbf{k} \cdot \mathbf{s}_{\mathbf{k}}-\sum_{\mathbf{k} \neq \mathbf{k}^{\prime}} \frac{V_{\mathbf{k}-\mathbf{k}^{\prime}}}{4 A} \mathbf{s}_{\mathbf{k}} \cdot \mathbf{s}_{\mathbf{k}^{\prime}},
$$

where $\mathbf{s}_{\mathbf{k}}=\sum_{\sigma, \sigma^{\prime}} \psi_{\mathbf{k}, \sigma}^{\dagger} \boldsymbol{\sigma}_{\sigma \sigma^{\prime}} \psi_{\mathbf{k}, \sigma^{\prime}}^{\dagger}$ is a spin operator for the $\mathbf{k}$ site of the momentum lattice. The first term in Eq. (4) is
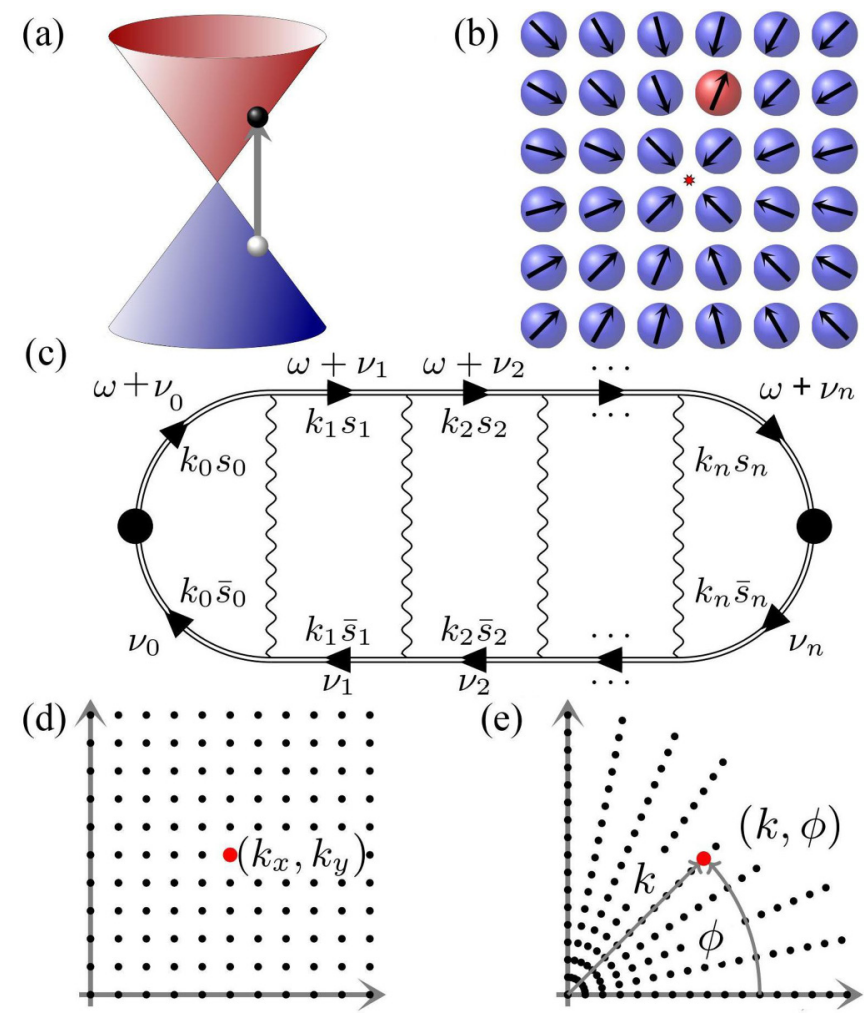

FIG. 1. (a),(b) Creation of an electron-hole pair as the flipping of a pseudospin on a vortex configuration. (c) $n$ th-order diagram of the KB particle-hole propagator associated with SCHF. Double lines are Green's functions dressed by SCHF self-energies, and wiggly lines are interaction matrices. (d),(e) Rediscretization from square to polar lattice.

a Zeeman vortex field and the second term is a long-range exchange coupling. This Hamiltonian is not exactly solvable but the fluctuations around the noninteracting state can be described by a Holstein-Primakoff expansion [25]. To do so, we choose a spin basis that diagonalizes the kinetic energy at each momentum site $\mathbf{S}_{\mathbf{k}}=-s_{\mathbf{k}}^{z} \hat{\mathbf{k}}+s_{\mathbf{k}}^{x} \hat{\mathbf{z}}+s_{\mathbf{k}}^{y} \hat{\boldsymbol{\phi}}$ where $\hat{\mathbf{z}}$ is the out-of-plane direction and $\hat{\boldsymbol{\phi}}=\hat{\mathbf{z}} \times \hat{\mathbf{k}}$. The spin operators can be expanded as $s_{\mathbf{k}}^{z} \approx 1-2 b_{\mathbf{k}}^{\dagger} b_{\mathbf{k}}^{\dagger}, s_{\mathbf{k}}^{x} \approx b_{\mathbf{k}}^{\dagger}+b_{\mathbf{k}}^{\dagger}$, and $i s_{\mathbf{k}}^{y} \approx$ $b_{\mathbf{k}}^{\dagger}-b_{\mathbf{k}}^{\dagger}$. Up to boson bilinears the Hamiltonian becomes (see $\S A 2$ of [26])

$$
H_{H P}=\sum_{\mathbf{k}, \mathbf{k}^{\prime}} B_{\mathbf{k}}^{\dagger} H_{\mathbf{k} \mathbf{k}^{\prime}} B_{\mathbf{k}^{\prime}},
$$

with $B_{\mathbf{k}}^{\dagger}=\left(b_{\mathbf{k}}^{\dagger} \quad b_{\mathbf{k}}^{\dagger}\right)$, and

$$
H_{\mathbf{k k}^{\prime}}=\delta_{\mathbf{k k}^{\prime}}\left(\begin{array}{cc}
2 E_{\mathbf{k}} & 0 \\
0 & -2 E_{\mathbf{k}}
\end{array}\right)-T_{\mathbf{k k}^{\prime}}
$$

with $\quad E_{\mathbf{k}}=v|\mathbf{k}|+\Sigma_{\mathbf{k}}, \quad \Sigma_{\mathbf{k}}=\sum_{\mathbf{k}^{\prime}} V_{\mathbf{k}-\mathbf{k}^{\prime}} \cos \phi_{\mathbf{k k}^{\prime}} / 2 A \quad$ is the Hartree-Fock self-energy, and $T_{\mathbf{k k}^{\prime}}$ is (for details see $\S A 1$ of [26])

$$
T_{\mathbf{k} \mathbf{k}^{\prime}}=\frac{V_{\mathbf{k}-\mathbf{k}^{\prime}}}{4 A}\left(\begin{array}{ll}
1+\cos \phi_{\mathbf{k} \mathbf{k}^{\prime}} & 1-\cos \phi_{\mathbf{k} \mathbf{k}^{\prime}} \\
1-\cos \phi_{\mathbf{k} \mathbf{k}^{\prime}} & 1+\cos \phi_{\mathbf{k} \mathbf{k}^{\prime}}
\end{array}\right) .
$$

Connection with perturbation theory. We will now demonstrate that the solution of the boson bilinear Hamiltonian 
in Eq. (5) is exactly equivalent to the calculation of the particle-hole propagator within the Kadanoff-Baym (KB) resummation of Feynman diagrams associated with the self-consistent Hartree-Fock (SCHF) approximation to the single-particle Green's function. In terms of electrons, the boson creation operator $b_{\mathbf{k}}^{\dagger}$ corresponds to the interband $\mathbf{Q}=0$ electron-hole pair creation operator: $b_{\mathbf{k}}^{\dagger}=\psi_{\mathbf{k}+}^{\dagger} \psi_{\mathbf{k}-\text {, }}^{\dagger}$, where the subindex $s= \pm$ denotes the conduction and valence bands. Therefore, our goal is to compute the electron-hole pair propagator defined as

$$
\chi_{\mathbf{k}_{1} \mathbf{k}_{2}}^{s_{1} s_{2}}(t)=-i T\left\langle\psi_{\mathbf{k}_{1} s_{1}}^{\dagger}(t) \psi_{\mathbf{k}_{1} \bar{s}_{1}}^{\dagger}(t) \psi_{\mathbf{k}_{2} \bar{s}_{2}}^{\dagger} \psi_{\mathbf{k}_{2} s_{2}}\right\rangle
$$

The KB SCHF resummation includes the entire BetheSalpeter ladder series with all internal single-particle Green's functions dressed with the SCHF self-energy [8,9]. These SCHF Green's functions are given by (see $\S A 3$ in [26])

$$
G_{\mathrm{k}}^{s_{1} s_{2}}(\omega)=\mathbf{k} s_{1} \longmapsto \mathrm{k}_{2}=\frac{\delta_{s_{1} s_{2}}}{\omega-s_{1}\left(E_{\mathbf{k}}-i \eta\right)} .
$$

and the $n$ th-order Feynman diagram of this series is shown in Fig. 1(c). The zeroth-order term of the series is

$$
\chi_{\mathbf{k}_{0} \mathbf{k}_{1}}^{(0)^{s_{0} s_{1}}}(\omega)=-\delta_{\mathbf{k}_{0} \mathbf{k}_{1}} \delta_{s_{0} s_{1}}\left(\frac{\delta_{s_{0}, 1}-\delta_{s_{0},-1}}{\omega-2 s_{0}\left(E_{\mathbf{k}_{0}}-i \eta\right)}\right) .
$$

An important property of this series, which can be readily obtained by integrating over internal intermediate frequencies, is that the intermediate Green's functions are all constrained to satisfy $s=s^{\prime}$, which physically means that the intermediate pairs always have one electron in the conduction band and a hole in the valence band. This allows one to cast the series as a matrix geometric series involving $\chi^{0}$ and $T$ of Eq. (7):

$$
\chi(\omega)=\chi^{0}(\omega)+\chi^{0}(\omega) T\left[\chi^{0}(\omega)+\chi^{0}(\omega) T \chi^{0}(\omega)+\cdots\right],
$$

and therefore the solution of the series has the form

$$
\chi_{\mathbf{k}_{0} \mathbf{k}_{f}}^{-1}(\omega)=-(\omega-i \eta) \tau^{z} \delta_{\mathbf{k}_{0} \mathbf{k}_{f}}-H_{\mathbf{k}_{0} \mathbf{k}_{f}},
$$

where $H_{\mathbf{k}_{0} \mathbf{k}_{f}}$ is given in Eq. (6) and $\tau^{z}$ is the diagonal Pauli matrix. The structure of this correlator is equal to the propagator of the Holstein-Primakoff (HP) bosons of the Hamiltonian (6). We therefore see that the exciton propagator has an identical effective Hamiltonian to the one obtained from the HP bosonic Hamiltonian in Eq. (6), demonstrating that the bosonized Hamiltonian is equivalent to self-consistent $\mathrm{KB}$ resummation of the particle-hole propagator.

Momentum space reparametrization. So far we have imagined our system to have a finite size so that momenta belongs to a discrete lattice. However, it is convenient to perform a reparametrization that manifestly displays the symmetries of the thermodynamic limit. If we parametrize momentum space by a new coordinate $\mathbf{z}(\mathbf{k})$, we can trade our boson Hamiltonian by one in a different lattice given by

$$
H_{H P}=\sum_{\mathbf{z}, \mathbf{z}^{\prime}} B_{\mathbf{z}}^{\dagger} H_{\mathbf{z z}} B_{\mathbf{z}^{\prime}} .
$$

As detailed in [26], in order to preserve the underlying microscopic normalization of the states, the boson operators and the Hamiltonian in the new lattice need to be rescaled as follows:

$$
B_{\mathbf{z}}=J(\mathbf{z}) B_{\mathbf{k}}, \quad H_{\mathbf{z z}^{\prime}}=J(\mathbf{z}) J\left(\mathbf{z}^{\prime}\right) H_{\mathbf{k k}^{\prime}},
$$

where $J(\mathbf{z})=\sqrt{D(\mathbf{z})\left(\Delta z_{1} \Delta z_{2}\right) /\left(\Delta k_{1} \Delta k_{2}\right)}, \Delta k_{i}=2 \pi / L_{i}, \Delta z_{i}$ is the discretization unit of the new coordinate system, and $D(\mathbf{z})$ is the Jacobian of the transformation. In particular, in order to exploit the emergent rotational invariance in the thermodynamic limit, we use the following polar parametrization $\mathbf{z}=(k, \phi)$ :

$$
k_{m}=\frac{\mathcal{K}}{\sqrt{2}} \tan ^{2}(m \Delta \theta), \quad \phi_{n}=n \Delta \phi,
$$

where $\left(k_{m}, \phi_{n}\right)$ are the polar coordinates of a given site in the polar momentum lattice depicted in Figs. 1(d) and 1(e), $\mathcal{K}$ is the UV momentum scale, $\Delta \theta=(\pi / 2) /(M+1), \Delta \phi=$ $2 \pi /(2 L+1)$, and $n=0, \ldots, 2 L, m=1, \ldots, M$.

The radial discretization we are choosing is denser at small $k$ and more dilute at large $k$. This is not crucial but allows faster numerical convergence at low energies. We have verified that the results we will describe are independent of the specific choice of the radial discretization once the grids become sufficiently dense [26].

Applying the transformation from Eq. (13) to the boson Hamiltonian from Eq. (12) leads to the following decoupling into angular momentum channels:

$$
B_{m}^{\ell}=\sum_{\ell=-L}^{L} e^{i \ell \phi_{n}} B_{m n}, \quad H_{H P}=\sum_{m \ell} B_{m}^{\ell \dagger} H_{m m^{\prime}}^{\ell} B_{m^{\prime}}^{\ell} .
$$

Therefore the problem reduces to a set of bosons moving in an effective one-dimensional radial space for each angular momentum channel which in general needs to be solved numerically.

Optical conductivity. As a concrete application of our formalism we study the Coulomb interaction corrections to the optical conductivity of Dirac fermions. We follow the Kubo approach to compute the conductivity from the current-current correlator $\chi_{\mu \nu}(t)=i \Theta(t) A\left\langle\left[j_{\mu}(t), j_{v}(0)\right]\right\rangle$. The total current operator carries $\mathbf{Q}=0$, so it can be represented exactly within the effective spin-1/2 Hilbert space of Eq. (4) as follows:

$$
\mathbf{j}=\frac{v}{A} \sum_{\mathbf{k}} \psi_{\mathbf{k} \sigma_{1}}^{\dagger} \sigma_{\sigma_{1} \sigma_{2}} \psi_{\mathbf{k} \sigma_{2}}=\frac{v}{A} \sum_{\mathbf{k}} \hat{\mathbf{s}}_{\mathbf{k}} .
$$

Using the HP approximation for the spin operators, the current-current correlator then can be expressed as (see Eq. (C8) in [26])

$$
\chi_{\varphi \varphi}(t)=i \Theta(t) \frac{2 v^{2} \Delta \theta}{(2 \mathcal{K})^{2}(2 \pi)} \sum_{m m^{\prime}} S_{m}\left\langle\left[B_{m}^{1 \dagger}(t), B_{m^{\prime}}^{1 \dagger}\right]\right\rangle S_{m^{\prime}},
$$

where $\left[B_{m}^{\ell \dagger}\right]=\left(\begin{array}{llllll}b_{0}^{\ell \dagger} & \cdots & b_{M}^{\ell \dagger} & b_{0}^{\ell \dagger} & \cdots & b_{M}^{\ell \dagger}\end{array}\right)$ and $\left[S_{m}\right]=$ $\left(t_{0} \cdots t_{M}-t_{0} \cdots-t_{M}\right)$ are scale factors with $t_{m}=$ $\sqrt[3]{\tan \left(\theta_{m}\right)} \sec \left(\theta_{m}\right)$. Because the current transforms as a vector under rotations, the calculation of the conductivity only requires solving the boson bilinear Hamiltonian of Eq. (15) for the angular momentum channel $\ell=1$, which is diagonalized by a transformation of the form (see [27])

$$
B_{m}^{1 \dagger}=\sum_{n} R_{m n}^{\dagger} D_{n}^{\dagger}, H_{m m^{\prime}}^{1}=\sum_{n n^{\prime}} R_{m n}^{*} \Omega_{n n^{\prime}} R_{n^{\prime} m^{\prime}}^{*}
$$



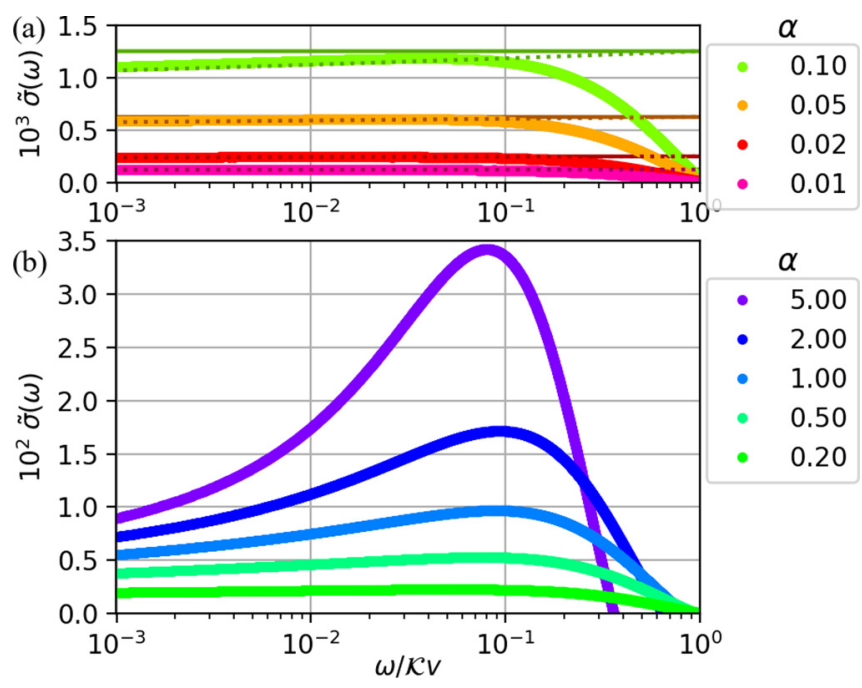

FIG. 2. (a) Conductivity at weak coupling. Thick: Bosonization approach. Dotted: Leading RG correction $\tilde{\sigma}(\omega)$. Solid: Leading perturbative correction from Ref. [12]. (b) Conductivity at strong coupling from the bosonization approach.

where $\Omega_{n n^{\prime}}=\operatorname{diag}\left(\omega_{0} \cdots \omega_{M}-\omega_{0} \cdots-\omega_{M}\right)$ is the diagonal matrix of the eigenvalues of Eq. (5). The real part of the conductivity can be obtained from the following Lehmanntype representation [see Eq. (C12) in [26]]:

$$
\sigma(\omega)=\frac{v^{2} \Delta \theta}{(2 \mathcal{K})^{2}} \sum_{m}\left|\sum_{n} R_{m n}^{*} S_{n}\right|^{2} \frac{\delta\left(\omega-\omega_{m}\right)}{\omega_{m}} .
$$

We will now describe the results for the optical conductivity obtained by numerically diagonalizing the $\ell=1$ angular momentum bilinear Hamiltonian of Eq. (15) for the Coulomb interaction $V_{\mathbf{q}}=2 \pi e^{2} / \epsilon|\mathbf{q}|$. Further details can be found in [26]. To isolate the interaction corrections to $\sigma(\omega)$ we define

$$
\tilde{\sigma}(\omega)=\frac{\sigma(\omega)-\sigma_{0}}{\sigma_{0}}
$$

where $\sigma_{0}=e^{2} / 16$ is the noninteracting conductivity of Dirac fermions. The leading perturbative correction to this conductivity is expected to be of the form [12] $\tilde{\sigma}=C \alpha+O\left(\alpha^{2}\right)$, with $C=(19-6 \pi) / 12$. We have been able to reproduce this perturbative correction numerically at small $\alpha$ as shown by solid horizontal lines in Fig. 2(a) along with the full numerical result from Eq. (18). At larger values of $\alpha$, clear deviations from the leading perturbative result are seen in Fig. 2(b). One of the conspicuous deviations is a logarithmic decrease of the conductivivity at low frequencies (see [26]). This logarithmic decrease can be explained by the logarithmic running of the coupling constant at small frequencies expected from the perturbative renormalization group (RG) analysis:

$$
\tilde{\sigma}(\omega)=\frac{C \alpha}{1+\frac{\alpha}{4} \ln \left(\frac{\mathcal{K} v}{\omega}\right)} \approx C \alpha\left[1+\frac{\alpha}{4} \ln \left(\frac{\omega}{\mathcal{K} v}\right)\right] .
$$

The predicted RG logarithmic correction is shown by a dotted line in Fig. 2(a), which is in good agreement with the numerical implementation of Eq. (20) at small $\alpha$. For larger values of $\alpha$ we see clear deviations from this leading RG perturbative result, as shown in Fig. 2(b).
Nevertheless, as shown in Fig. 2, even for a value of $\alpha$ as large as $\alpha=5$ the maximal deviation of the conductivity from the noninteracting value is only about $4 \%$. This indicates a resilience of conductivity of Dirac fermions to interaction corrections even when nonpertubative effects are included, in agreement with experiments that have obtained values close to those of noninteracting fermions [22-24]. We would like to note that our formalism so far neglects the role of screening [namely, the wavy interaction lines in Fig. 1(a) are the bare Coulomb interactions]. The qualitative role of screening is expected to be secondary since the system has a vanishing density of states. Nevertheless, screening can play an important role quantitatively at strong coupling, and we actually expect that the interaction corrections to the optical conductivity upon including screening would be even smaller than the corrections we obtained, because within the RPA screening should lead to a reduction of the effective value of $\alpha \rightarrow \alpha_{\mathrm{RPA}} \approx \alpha /(1+\pi N \alpha / 8)$, where $N$ is the total number of Dirac cones (e.g., $N=4$ for graphene [16]). We note that a challenge in systematically accounting for the coupling of the modes at $\mathbf{Q}=0$ that we have studied to the density fluctuation modes at nonzero $\mathbf{Q}$, which are responsible for screening, is that, because of momentum conservation, such coupling would necessarily include boson cubic and higherorder nonlinear terms in the effective bosonic Hamiltonian, making it challenging to solve the problem in an essentially exact manner as we have so far done.

Discussion and summary. We have developed a formalism that captures nonperturbatively the interaction effects on the continuum of $\mathbf{Q}=0$ particle-hole excitations of Dirac fermions. Our approach is constructed by projecting the full microscopic many-body Hamiltonian of Dirac fermions into the subspace of singly occupied momentum states, leading to an effective spin-1/2 Heisenberg-like model in a momentum lattice. This problem is subsequently reduced to a boson bilinear Hamiltonian by a standard Holstein-Primakoff transformation. We have provided a solid microscopic justification for this formalism by showing that it is equivalent to the Kadanoff-Baym resummation of the particle-hole propagator associated with the SCHF approximation to the singleparticle Green's function. This approximation is expected to capture the essential universal low-energy properties of the semimetallic phase that evolves adiabatically from free fermions. We have applied this formalism to compute the Coulomb interaction corrections to the optical conductivity of Dirac fermions and found that it recovers the results of perturbative renormalization group at weak coupling [12] and extended them to strong coupling. Remarkably, we have found that the Coulomb interaction corrections remain very weak $(\sim 4 \%)$ up to values of the effective fine-structure constant $\alpha \sim 5$, in agreement with experiments in graphene that have measured a value of the optical conductivity that is consistent with the free-electron theory [22-24]. Although our discussion has been restricted to 2D Dirac fermions, our approach can be naturally generalized to other multiband semimetals and higher dimensions, such as Weyl semimetals [28] and novel nodal fermions [29], providing an interesting tool to capture nonperturbative effects of interactions on the correlation functions of $\mathbf{Q}=0$ operators of these phases. 
[1] T. Giamarchi, Quantum Physics in One Dimension (Clarendon, Oxford, 2003), Vol. 121.

[2] A. Luther, Tomonaga fermions and the Dirac equation in three dimensions, Phys. Rev. B 19, 320 (1979).

[3] F. Haldane, Luttinger's Theorem and Bosonization of the Fermi Surface, in Proceedings of the International School of Physics "E. Fermi", edited by R. A. Broglia and J. R. Schrieffer, Course CXXI, Perspectives in Many-Particle Physics (North-Holland, Amsterdam, 1994), pp. 5-29, arXiv:cond-mat/0505529.

[4] A. Houghton and J. B. Marston, Bosonization and fermion liquids in dimensions greater than one, Phys. Rev. B 48, 7790 (1993).

[5] A. H. Castro Neto and E. Fradkin, Bosonization of the Low Energy Excitations of Fermi Liquids, Phys. Rev. Lett. 72, 1393 (1994).

[6] A. Houghton, H.-J. Kwon, and J. Marston, Multidimensional bosonization, Adv. Phys. 49, 141 (2000).

[7] A. H. Castro Neto and E. H. Fradkin, Exact solution of the Landau fixed point via bosonization, Phys. Rev. B 51, 4084 (1995).

[8] G. Baym and L. P. Kadanoff, Conservation Laws and Correlation Functions, Phys. Rev. 124, 287 (1961).

[9] G. Baym, Self-Consistent Approximations in Many-Body Systems, Phys. Rev. 127, 1391 (1962).

[10] A. W. W. Ludwig, M. P. A. Fisher, R. Shankar, and G. Grinstein, Integer quantum Hall transition: An alternative approach and exact results, Phys. Rev. B 50, 7526 (1994).

[11] T. Ando, Y. Zheng, and H. Suzuura, Dynamical conductivity and zero-mode anomaly in honeycomb lattices, J. Phys. Soc. Jpn. 71, 1318 (2002).

[12] E. Mishchenko, Minimal conductivity in graphene: Interaction corrections and ultraviolet anomaly, Europhys. Lett. 83, 17005 (2008).

[13] I. F. Herbut, V. Juričić, and O. Vafek, Coulomb Interaction, Ripples, and the Minimal Conductivity of Graphene, Phys. Rev. Lett. 100, 046403 (2008).

[14] D. E. Sheehy and J. Schmalian, Optical transparency of graphene as determined by the fine-structure constant, Phys. Rev. B 80, 193411 (2009).

[15] S. H. Abedinpour, G. Vignale, A. Principi, M. Polini, W.-K. Tse, and A. H. MacDonald, Drude weight, plasmon dispersion, and ac conductivity in doped graphene sheets, Phys. Rev. B 84, 045429 (2011).

[16] I. Sodemann and M. M. Fogler, Interaction corrections to the polarization function of graphene, Phys. Rev. B 86, 115408 (2012).
[17] G. Gazzola, A. Cherchiglia, L. Cabral, M. Nemes, and M. Sampaio, Conductivity of Coulomb interacting massless Dirac particles in graphene: Regularization-dependent parameters and symmetry constraints, Europhys. Lett. 104, 27002 (2013).

[18] E. Barnes, E. H. Hwang, R. E. Throckmorton, and S. Das Sarma, Effective field theory, three-loop perturbative expansion, and their experimental implications in graphene many-body effects, Phys. Rev. B 89, 235431 (2014).

[19] S. Teber and A. Kotikov, Interaction corrections to the minimal conductivity of graphene via dimensional regularization, Europhys. Lett. 107, 57001 (2014).

[20] S. Teber and A. Kotikov, Field theoretic renormalization study of interaction corrections to the universal ac conductivity of graphene, J. High Energy Phys. 07 (2018) 82.

[21] D. L. Boyda, V. V. Braguta, M. I. Katsnelson, and M. V. Ulybyshev, Many-body effects on graphene conductivity: Quantum Monte Carlo calculations, Phys. Rev. B 94, 085421 (2016).

[22] Z. Li, E. A. Henriksen, Z. Jiang, Z. Hao, M. C. Martin, P. Kim, H. Stormer, and D. N. Basov, Dirac charge dynamics in graphene by infrared spectroscopy, Nat. Phys. 4, 532 (2008).

[23] K. F. Mak, M. Y. Sfeir, Y. Wu, C. H. Lui, J. A. Misewich, and T. F. Heinz, Measurement of the Optical Conductivity of Graphene, Phys. Rev. Lett. 101, 196405 (2008).

[24] R. R. Nair, P. Blake, A. N. Grigorenko, K. S. Novoselov, T. J. Booth, T. Stauber, N. M. Peres, and A. K. Geim, Fine structure constant defines visual transparency of graphene, Science $\mathbf{3 2 0}$, 1308 (2008).

[25] A. Auerbach, Interacting Electrons and Quantum Magnetism (Springer Science \& Business Media, Berlin, 2012).

[26] See Supplemental Material at http://link.aps.org/supplemental/ 10.1103/PhysRevB.102.121103 for derivations of the expressions.

[27] J. Van Hemmen, A note on the diagonalization of quadratic boson and fermion Hamiltonians, Z. Phys. B 38, 271 (1980).

[28] N. P. Armitage, E. J. Mele, and A. Vishwanath, Weyl and Dirac semimetals in three-dimensional solids, Rev. Mod. Phys. 90, 015001 (2018).

[29] B. Bradlyn, J. Cano, Z. Wang, M. Vergniory, C. Felser, R. J. Cava, and B. A. Bernevig, Beyond Dirac and Weyl fermions: Unconventional quasiparticles in conventional crystals, Science 353, aaf5037 (2016). 